\title{
NOTA/NOTE
}

\section{Abundancia del pez león invasor Pterois volitans en los arrecifes de coral costeros del Caribe colombiano}

\section{Abundance of the invasive lionfish Pterois volitans in the coastal coral reefs from Colombian Caribbean}

\author{
Luis Chasqui*, Natalia Rincón-Díaz y María Juliana Vanegas \\ (D) $0000-0002-9381-2301 \quad$ (D) $0000-0002-4908-4802 \quad$ (D) $0000-0002-0125-0728$ \\ 1. Instituto de Investigaciones Marinas y Costeras (INVEMAR), Santa Marta, Colombia.natalia.rincon@invemar.org.co,majuli_19@hotmail.com, \\ luis.chasqui@invemar.org.co*Autor para correspondencia.
}

\section{RESUMEN}

$\mathrm{S}$ e evaluó la abundancia del pez león invasor Pterois volitans mediante censos visuales realizados en 2015 y 2016 en 54 sitios de seis localidades con arrecifes de coral a lo largo de la costa Caribe colombiana. El invasor se observó en todos los lugares y en la mayoría de las inmersiones, con una frecuencia de avistamiento de $88 \%$, valores de abundancia desde 0 hasta $9,7 \pm 2,1 \mathrm{ind} / 250 \mathrm{~m}^{2}$ (388 ind/ha) y un promedio de $0,8 \pm 1,3 \mathrm{ind} / 250 \mathrm{~m}^{2}$ (32 ind/ha). La densidad media fue similar a los valores reportados en otros trabajos que contaron pez león en una amplia escala espacial en el área invadida y el valor más alto se aproximó a la densidad máxima estimada en Colombia y otros lugares del Caribe.

PALABRAS CLAVE: Caribe sur, especie exótica invasora, censos visuales submarinos, densidad de pez león.

\section{ABSTRACT}

The abundance of the invasive lionfish Pterois volitans was assessed through visual censuses conducted in 2015 and 2016 at 54 sites in six localities with coral reefs across the Colombian Caribbean coast. The invader was observed across locations and in most of the dives, with sighting frequency of $88 \%$, abundance values from 0 to $9.7 \pm 2.1 \mathrm{ind} / 250 \mathrm{~m}^{2}(388 \mathrm{ind} / \mathrm{ha})$, and $0.8 \pm 1.3 \mathrm{ind} / 250 \mathrm{~m}^{2}$ (32 ind/ha) as total mean. The average density was similar to values reported in other works that counted lionfish in a broad spatial scale on the invaded area and the highest value approximated the maximum density estimated in Colombia and other locations around the Caribbean.

KEY WORDS: southern Caribbean, invasive alien species, underwater visual census, lionfish density. 
El pez león Pterois volitans (Linnaeus) es un escorpénido nativo del Indopacífico que fue introducido en el Atlántico occidental ( $\mathrm{AO}$ ) hace más de tres décadas. La primera captura conocida de la especie en el AO tuvo lugar en Florida, EE. UU., en 1985, y en la década siguiente se conocieron registros adicionales en lugares de EE. UU. y las Bermudas (Schofield, 2009). Para el 2010, el pez león ya se consideraba una especie exótica invasora en la mayor parte del gran Caribe, incluida Colombia (González et al., 2009; Schofield, 2010). Mostrando una notable capacidad de dispersión, el invasor en menos de 30 años hizo presencia en gran parte del AO, desde Carolina del Norte en la costa este de los EE. UU., el golfo de México y el Caribe (USGSNAS, 2020). Con un área ocupada de casi 7,3 millones de $\mathrm{km}^{2}$ (Côté et al., 2013), la invasión del pez león es ahora reconocida como la más rápida por un pez marino en la historia (Hamner et al., 2007; Schofield, 2010; Betancur-R et al., 2011). Incluso hay un registro de un pez león capturado en 2014 tan al sur como lo es Río de Janeiro, Brasil (Ferreira et al., 2015).

El pez león posee un conjunto de características biológicas que determinan su éxito como invasor y lo convierten en una seria amenaza para la biota nativa del AO: un rápido crecimiento (Pusack et al., 2016) y un alto rendimiento reproductivo (Gardner et al., 2015; Fogg et al., 2017), así como rasgos generalistas en hábitat y dieta (Muñoz et al., 2011; Claydon et al., 2012; Cure et al., 2014; Acero et al., 2019); además de una serie de características fisiológicas, morfológicas y de comportamiento que lo convierten en un cazador poderoso y en una presa difícil (Morris, 2013; DíazFerguson y Hunter, 2019; Green et al., 2019; Rojas et al., 2019). La aparente ubicuidad y la abundancia de la especie en el área invadida, en sinergia con su carácter de depredador voraz y oportunista, le dan un gran potencial para afectar catastróficamente las comunidades de peces nativos (Côté et al.,2013; Ballew et al., 2016; Ingeman, 2016). Las poblaciones de peces en los arrecifes de coral están particularmente en riesgo de descenso por el pez león, poniendo en peligro la economía local de los pescadores (Albins y Hixon, 2008; Green et al., 2012) e incluso provocando efectos en cascada en la estructura de las comunidades bentónicas (Lesser y Slattery, 2011).

El primer avistamiento registrado de pez león en Colombia data de diciembre de 2008 en el sitio de buceo Manta City, en la isla de Providencia, a $5 \mathrm{~m}$ de profundidad (Schofield, 2009). Cinco meses después, la especie se registró en el Parque Nacional Natural (PNN) Tayrona a 16 m
The lionfish Pterois volitans (Linnaeus) is a scorpionfish native to the Indo-Pacific that was introduced in the Western Atlantic (WA) more than three decades ago. The first known capture of the species in the WA took place in Florida, USA, in 1985, and in the following decade additional records from locations in the USA and Bermuda were known (Schofield, 2009). By 2010 the lionfish was already considered an exotic invasive species across most of the Greater Caribbean, including Colombia (González et al., 2009; Schofield, 2010). Showing a remarkable dispersal capacity, in less than 30 years the invader made presence in most of the WA, from North Carolina on the east coast of USA, Gulf of Mexico, and the Caribbean (USGS-NAS, 2020). With an occupied area of almost 7.3 million $\mathrm{km}^{2}$ (Côté et al., 2013), lionfish invasion is now recognized as the fastest invasion by a marine fish in history (Hamner et al., 2007; Schofield, 2010; Betancur-R et al., 2011). There is even a report of a lionfish caught in 2014 as far south as Rio de Janeiro, Brazil (Ferreira et al., 2015).

The lionfish has a set of biological characteristics that determine its success as an invader and turn it on a serious threat to the WA native biota: a rapid growth (Pusack et al., 2016) and high reproductive output (Gardner et al., 2015; Fogg et al., 2017), as well as generalist traits in habitat and diet (Muñoz et al., 2011; Claydon et al., 2012; Cure et al., 2014; Acero et al., 2019); in addition to a series of physiological, morphological and behavioral characteristics make it a mighty hunter and a difficult prey (Morris, 2013; Díaz-Ferguson and Hunter, 2019; Green et al., 2019; Rojas et al., 2019). The apparent everywhereness and abundance of the species in the invaded area synergistically with its character as a voracious and opportunistic predator, give it a great potential to catastrophically affect the native fish communities (Côté et al., 2013; Ballew et al., 2016; Ingeman, 2016). Coral reefs fish populations are particularly in risk of reduction by lionfish, putting local fishermen's economy in peril (Albins and Hixon, 2008; Green et al., 2012) and even triggering cascading effects on the benthic community's structure (Lesser and Slattery, 2011).

The first recorded sighting of lionfish in Colombia dates of December 2008 at the Manta City dive site, on the island of Providencia, at $5 \mathrm{~m}$ depth (Schofield, 2009). Five months later the species was recorded in the Tayrona National Natural Park (NNP) at 16 m depth (González et al., 2009), and since then a lot of records have been received in the INVEMAR database for the entire 
de profundidad (González et al., 2009), y desde entonces se han recibido muchos registros de todo el Caribe colombiano en la base de datos del INVEMAR (http://invasoresmarinos. invemar.org.co/). Desde ese primer registro, se han adelantado en el país investigaciones sobre la invasión en varios temas, desde la caracterización de la especie (Hernández-Abello et al., 2015; González et al., 2011) hasta aspectos de su ecología trófica (e.g. Muñoz-Escobar y Gil-Agudelo, 2012; Pabón y Acero, 2015; Acero et al., 2019). Algunos estudios han estimado la abundancia del pez león en lugares específicos, como el PNN Tayrona y Santa Marta (Bayraktarov et al., 2014; Coronado-Carrascal et al., 2015; García-Urueña et al., 2015), y en la isla de San Andrés (González-Corredor et al., 2016), pero hasta la fecha, solo existe un trabajo publicado en el que se evaluaron lugares distantes (Rojas-Vélez et al., 2019). Hasta el momento, no hay estudios de la abundancia de esta especie con metodologías estandarizadas y en un número de localidades que permitan comparaciones en una escala espacial más amplia. En ese sentido, este trabajo ofrece información sobre la distribución y abundancia del pez león a nivel regional en Colombia, contribuyendo al acervo de información sobre esta invasión biológica en el país.

Entre agosto-octubre de 2015 y mayo-septiembre de 2016 se visitaron las seis localidades con la mayor extensión de formaciones coralinas en el Caribe continental colombiano, a fin de verificar la presencia y estimar la abundancia del pez león (Figura 1; Tabla 1). Los censos de pez león se hicieron entre las 7 a. m. y las 5 p. m. siguiendo la metodología de Green (2013), ampliamente utilizada en el AO. Mediante buceo SCUBA se realizó una búsqueda intensiva del pez león en transectos de $25 \times 10 \mathrm{~m}$, cuidando de buscar bajo las salientes y entre las grietas y oquedades del arrecife.

Los valores de densidad se estimaron en individuos por $250 \mathrm{~m}^{2}$, que se promediaron por sitio y localidad $\mathrm{y}$ se extrapolaron a hectáreas para comparación con otros estudios. El número de censos fue variable entre sitios (3 a 12) (Tabla 1) porque el tiempo para evaluar cada transecto cambió de acuerdo con los rasgos físicos de los sitios de muestreo (e.g. mayor/menor complejidad estructural, profundidad, visibilidad); así como con el tiempo de buceo en línea con los límites de descompresión del buceo con aire. Para evaluar las diferencias de densidad entre años, entre ubicaciones y en relación con la profundidad, se utilizó la prueba estadística para medianas de Mood. Previamente, se analizó la normalidad y la homocedasticidad de los datos con las pruebas de Shapiro-Wilk y Bartlett. Para los análisis, se clasificaron las profundidades así: 1: 1-10 m, 2: > 10-20 m,
Colombian Caribbean (http://invasoresmarinos.invemar. org.co/). Since the first record, research on the invasion has been carried out in the country in several topics, from the characterization of the species (Hernández-Abello et al., 2015; González et al., 2011) to its trophic ecology (e.g. Muñoz-Escobar and Gil-Agudelo, 2012; Pabón and Acero, 2015; Acero et al., 2019). Some studies have estimated lionfish abundance on specific locations, such as Tayrona NNP and Santa Marta (Bayraktarov et al., 2014; CoronadoCarrascal et al., 2015; García-Urueña et al., 2015), and San Andrés island (González-Corredor et al., 2016); but until date, just one published work exists in which distant locations were evaluated (Rojas-Vélez et al., 2019). So far, there are no reports of this species abundance with standardized methodologies and in a number of locations as to make comparisons on a broader spatial scale. In that sense, this work offers information on the distribution and abundance of the lionfish on a regional level in Colombia, contributing to the information pool on this biological invasion in the country.

Between August-October 2015 and MaySeptember 2016, the six localities with the most significant extension of coral formations throughout the Colombian continental Caribbean were visited to verify the presence and estimate the abundance of lionfish (Figure 1; Table 1). Lionfish census were made between $7-17 \mathrm{~h}$ following the Green (2013) methodology, widely used in the WA. Through SCUBA diving, an intensive search of lionfish in transects of $25 \times 10 \mathrm{~m}$ was carried out, taking care of searching under the overhangs and among reef cavities and cracks.

Density values were estimated in individuals per $250 \mathrm{~m}^{2}$, averaged by site and locality and extrapolated to hectares for comparison with other studies. The number of census vary between sites ( 3 to 12 ) (Table 1 ) because time to evaluate each transect changed according to physical traits of the sampling sites (e.g. greater/lesser structural complexity, depth, visibility); as well as with diving time in line to decompression limits of scuba diving with air. To assess for differences in density among years, between locations, and related to depth, the statistical test of Mood for the medians was used. Previously, the normality and homoscedasticity of the data were analyzed with the Shapiro-Wilk and Bartlett tests. For the analyzes depth were ranked as follows: 1: 1-10 m, 2: > 10-20 m, 3: > 20-30 m, 4: $>30-40 \mathrm{~m}$. All analyzes were done with Statgraphics Centurion XVI. 


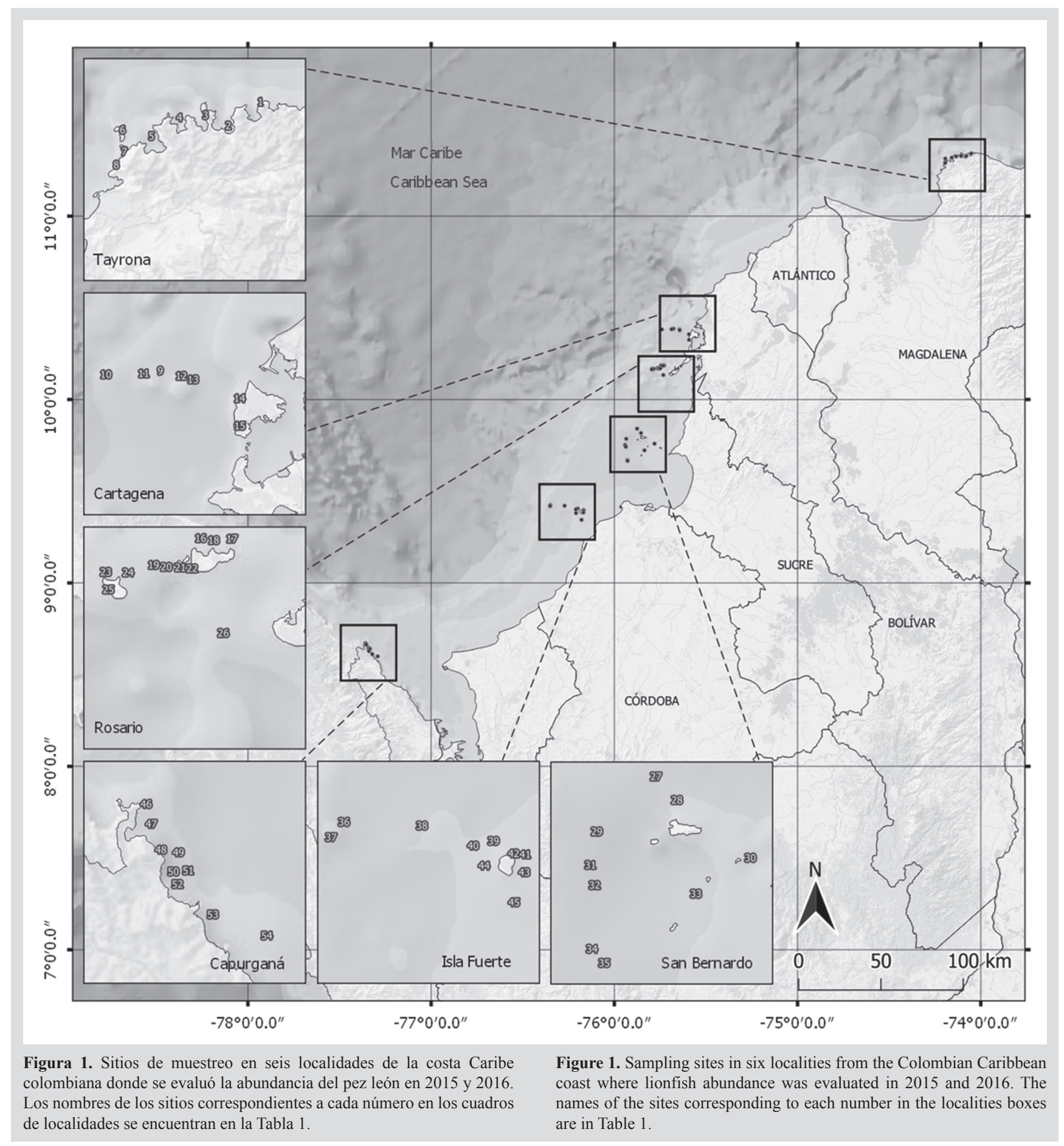

\section{3: > 20-30 m, 4: > 30-40 m. Todos los análisis se hicieron con} Statgraphics Centurion XVI.

Se hicieron en total 452 censos en 54 sitios en las seis localidades durante los dos años (191 en 2015; 261 en 2016), cubriendo un área muestreada total de $113000 \mathrm{~m}^{2}$ entre 4,5 y $44 \mathrm{~m}$ de profundidad. La búsqueda en cada transecto duró 17,3 min en promedio para un tiempo total de muestreo de alrededor de $130 \mathrm{~h}$, durante el cual se observaron 350
A total of 452 censuses were conducted in 54 sites in the six localities during the two years (191 in 2015 ; 261 in 2016), with a sampled area of $113,000 \mathrm{~m}^{2}$ between 4.5 and $44 \mathrm{~m}$ deep. The search in each transect lasted $17.3 \mathrm{~min}$ on average for a whole sampling time of around $130 \mathrm{~h}$, during which 350 lionfishes were observed in 185 transects from 48 sites. From a total of 85 dives, the invader was observed in 75 for a sighting frequency 
Tabla 1. Abundancia del pez león en la costa Caribe colombiana en 2015 y 2016. Densidad promedio (individuos $/ 250 \mathrm{~m}^{2}$ ) \pm 1 desviación estándar con el número de censos por sitio de muestreo entre paréntesis. ND significa que el sitio no fue evaluado ese año.

\begin{tabular}{|c|c|c|c|c|c|}
\hline Localidad/Locality & Sitio de muestreo/Samplin & site & Geolocalización/Geolocation & 2015 & 2016 \\
\hline \multirow{8}{*}{$\begin{array}{l}\text { Parque Nacional Natural Tayrona/ } \\
\text { Tayrona National Natural Park }\end{array}$} & Cinto & 1 & $11^{\circ} 20^{\prime} 31^{\prime \prime} \mathrm{N}, 74^{\circ} 03^{\prime} 10^{\prime \prime} \mathrm{W}$ & $0.8 \pm 0.8(6)$ & $1.2 \pm 2.0(6)$ \\
\hline & Neguanje & 2 & $11^{\circ} 19^{\prime} 42^{\prime \prime} \mathrm{N}, 74^{\circ} 04^{\prime} 48^{\prime \prime} \mathrm{W}$ & $0(6)$ & $0.4 \pm 0.8(12)$ \\
\hline & Gayraca & 3 & $11^{\circ} 19^{\prime} 42^{\prime \prime} \mathrm{N}, 74^{\circ} 06^{\prime} 33^{\prime \prime} \mathrm{W}$ & $0(6)$ & $0.5 \pm 0.6(4)$ \\
\hline & Chengue & 4 & $11^{\circ} 19^{\prime} 33^{\prime \prime} \mathrm{N}, 74^{\circ} 08^{\prime} 09^{\prime \prime} \mathrm{W}$ & $0.2 \pm 0.4(6)$ & $1.2 \pm 1.3(6)$ \\
\hline & Concha & 5 & $11^{\circ} 19^{\prime} 09^{\prime \prime} \mathrm{N}, 74^{\circ} 09^{\prime} 34^{\prime \prime} \mathrm{W}$ & $0.7 \pm 1.2(6)$ & $1.5 \pm 2.1(6)$ \\
\hline & Isla Aguja/Aguja island & 6 & $11^{\circ} 18^{\prime} 48^{\prime \prime} \mathrm{N}, 74^{\circ} 11^{\prime} 39^{\prime \prime} \mathrm{W}$ & ND & $0.8 \pm 1.6(6)$ \\
\hline & Granate & 7 & $11^{\circ} 17^{\prime} 50^{\prime \prime} \mathrm{N}, 74^{\circ} 11^{\prime} 33^{\prime \prime} \mathrm{W}$ & $1.0 \pm 1.1(6)$ & $0.3 \pm 0.5(6)$ \\
\hline & Punta Granate & 8 & $11^{\circ} 17^{\prime} 23^{\prime \prime} \mathrm{N}, 74^{\circ} 11^{\prime} 44^{\prime \prime} \mathrm{W}$ & ND & $0.2 \pm 0.4(6)$ \\
\hline \multirow{7}{*}{ Cartagena } & Salmedina & 9 & $10^{\circ} 23^{\prime} 12^{\prime \prime} \mathrm{N}, 75^{\circ} 40^{\prime} 40^{\prime \prime} \mathrm{W}$ & $1.2 \pm 2.2(5)$ & $0.8 \pm 1.2(6)$ \\
\hline & Burbujas 2 & 10 & $10^{\circ} 22^{\prime} 57^{\prime \prime} \mathrm{N}, 75^{\circ} 44^{\prime} 28^{\prime \prime} \mathrm{W}$ & $0.4 \pm 0.5(5)$ & ND \\
\hline & Burbujas Nati & 11 & $10^{\circ} 23^{\prime} 01^{\prime \prime} \mathrm{N}, 75^{\circ} 41^{\prime} 18^{\prime \prime} \mathrm{W}$ & $1.3 \pm 0.5(6)$ & $0.5 \pm 0.9(12)$ \\
\hline & Barco Faro & 12 & $10^{\circ} 22^{\prime} 57^{\prime \prime} \mathrm{N}, 75^{\circ} 38^{\prime} 43^{\prime \prime} \mathrm{W}$ & $0.7 \pm 1.2(6)$ & $1.8 \pm 2.1(6)$ \\
\hline & Barco Cemento & 13 & $10^{\circ} 22^{\prime} 43^{\prime \prime} \mathrm{N}, 75^{\circ} 38^{\prime} 41^{\prime \prime} \mathrm{W}$ & $1.2 \pm 0.8(5)$ & ND \\
\hline & Horno & 14 & $10^{\circ} 21^{\prime} 22^{\prime \prime} \mathrm{N}, 75^{\circ} 35^{\prime} 36^{\prime \prime} \mathrm{W}$ & $0.2 \pm 0.4(6)$ & ND \\
\hline & La Monja & 15 & $10^{\circ} 19^{\prime} 33^{\prime \prime} \mathrm{N}, 75^{\circ} 35^{\prime} 35^{\prime \prime} \mathrm{W}$ & ND & $1.0 \pm 0.9(6)$ \\
\hline \multirow{11}{*}{$\begin{array}{l}\text { Archipiélago del Rosario/ } \\
\text { Rosario archipelago }\end{array}$} & Punta Brava & 16 & $10^{\circ} 11^{\prime} 11^{\prime \prime} \mathrm{N}, 75^{\circ} 44^{\prime} 45^{\prime \prime} \mathrm{W}$ & ND & $0.5 \pm 0.8(6)$ \\
\hline & Isla Fiesta/Fiesta island & 17 & $10^{\circ} 11^{\prime} 10^{\prime \prime} \mathrm{N}, 75^{\circ} 43^{\prime} 43^{\prime \prime} \mathrm{W}$ & $0.9 \pm 1.0(10)$ & $0.2 \pm 0.4(6)$ \\
\hline & Latifundio & 18 & $10^{\circ} 11^{\prime} 07^{\prime \prime} \mathrm{N}, 75^{\circ} 44^{\prime} 08^{\prime \prime} \mathrm{W}$ & $1.2 \pm 1.3(5)$ & $1.5 \pm 1.6(6)$ \\
\hline & Pavitos & 19 & $10^{\circ} 10^{\prime} 18^{\prime \prime} \mathrm{N}, 75^{\circ} 45^{\prime} 56^{\prime \prime} \mathrm{W}$ & $0(5)$ & $0(6)$ \\
\hline & Alex Place & 20 & $10^{\circ} 10^{\prime} 14^{\prime \prime} \mathrm{N}, 75^{\circ} 45^{\prime} 42^{\prime \prime} \mathrm{W}$ & ND & $0.3 \pm 0.8(6)$ \\
\hline & Telber & 21 & $10^{\circ} 10^{\prime} 13^{\prime \prime} \mathrm{N}, 75^{\circ} 45^{\prime} 14^{\prime \prime} \mathrm{W}$ & ND & $0.5 \pm 0.8(6)$ \\
\hline & Luis Guerra & 22 & $10^{\circ} 10^{\prime} 11^{\prime \prime} \mathrm{N}, 75^{\circ} 45^{\prime} 02^{\prime \prime} \mathrm{W}$ & $0.8 \pm 0.8(5)$ & $0.2 \pm 0.4(6)$ \\
\hline & Rosario 2 & 23 & $10^{\circ} 10^{\prime} 04^{\prime \prime} \mathrm{N}, 75^{\circ} 47^{\prime} 42^{\prime \prime} \mathrm{W}$ & $0.4 \pm 0.5(5)$ & ND \\
\hline & Barco Hundido & 24 & $10^{\circ} 10^{\prime} 03^{\prime \prime} \mathrm{N}, 75^{\circ} 47^{\prime} 09^{\prime \prime} \mathrm{W}$ & $0(5)$ & $0.3 \pm 0.5(6)$ \\
\hline & Rosario 1 & 25 & $10^{\circ} 09^{\prime} 53^{\prime \prime} \mathrm{N}, 75^{\circ} 47^{\prime} 48^{\prime \prime} \mathrm{W}$ & $0.4 \pm 0.5(5)$ & ND \\
\hline & Isla Arena/Arena island & 26 & $10^{\circ} 08^{\prime} 03^{\prime \prime} \mathrm{N}, 75^{\circ} 44^{\prime} 00^{\prime \prime} \mathrm{W}$ & $0.6 \pm 1.3(5)$ & $0.7 \pm 1.2(6)$ \\
\hline \multirow{9}{*}{$\begin{array}{l}\text { Archipiélago de San Bernardo/ } \\
\text { San Bernardo archipelago }\end{array}$} & Bajo Picuas & 27 & $09^{\circ} 50^{\prime} 29^{\prime \prime} \mathrm{N}, 75^{\circ} 52^{\prime} 35^{\prime \prime} \mathrm{W}$ & ND & $0(4)$ \\
\hline & Bajo del Medio & 28 & $09^{\circ} 49^{\prime} 04^{\prime \prime} \mathrm{N}, 75^{\circ} 51^{\prime} 19^{\prime \prime} \mathrm{W}$ & ND & $0(4)$ \\
\hline & Bajo Tiosolda & 29 & $09^{\circ} 47^{\prime} 10^{\prime \prime} \mathrm{N}, 75^{\circ} 56^{\prime} 09^{\prime \prime} \mathrm{W}$ & ND & $0(5)$ \\
\hline & Isla Mangle/Mangle island & 30 & $09^{\circ} 45^{\prime} 35^{\prime \prime} \mathrm{N}, 75^{\circ} 46^{\prime} 53^{\prime \prime} \mathrm{W}$ & ND & $0(4)$ \\
\hline & Bajo Minalta 2 & 31 & $09^{\circ} 45^{\prime} 09^{\prime \prime} \mathrm{N}, 75^{\circ} 56^{\prime} 32^{\prime \prime} \mathrm{W}$ & ND & $0(4)$ \\
\hline & Bajo Minalta & 32 & $09^{\circ} 44^{\prime} 34^{\prime \prime} \mathrm{N}, 75^{\circ} 56^{\prime} 17^{\prime \prime} \mathrm{W}$ & ND & $0.8 \pm 0.8(5)$ \\
\hline & N20 & 33 & $09^{\circ} 43^{\prime} 25^{\prime \prime} \mathrm{N}, 75^{\circ} 50^{\prime} 10^{\prime \prime} \mathrm{W}$ & ND & $0.5 \pm 0.6(4)$ \\
\hline & Bajo Nuevo Norte & 34 & $09^{\circ} 40^{\prime} 11^{\prime \prime} \mathrm{N}, 75^{\circ} 55^{\prime} 46^{\prime \prime} \mathrm{W}$ & ND & $0.8 \pm 1.0(4)$ \\
\hline & Bajo Nuevo & 35 & $09^{\circ} 39^{\prime} 58^{\prime \prime} \mathrm{N}, 75^{\circ} 55^{\prime} 42^{\prime \prime} \mathrm{W}$ & ND & $1.0 \pm 1.2(4)$ \\
\hline
\end{tabular}

Table 1. Lionfish abundance in the Colombian Caribbean coast in 2015 the number of censuses per sampling site in parentheses. ND means the site and 2016. Average density (individuals $/ 250 \mathrm{~m}^{2}$ ) \pm 1 standard deviation with and 2016 . Average density (individuals $/ 250 \mathrm{~m}^{2}$ ) \pm 1 standard deviation with 


\begin{tabular}{|c|c|c|c|c|c|}
\hline Localidad/Locality & Sitio de muestreo/Sampling & site & Geolocalización/Geolocation & 2015 & 2016 \\
\hline \multirow{10}{*}{ Isla Fuerte/Fuerte island } & Bushnell-Laberinto & 36 & $09^{\circ} 25^{\prime} 29^{\prime \prime} \mathrm{N}, 76^{\circ} 21^{\prime} 00^{\prime \prime} \mathrm{W}$ & $2.8 \pm 2.5(4)$ & $0.3 \pm 0.6(3)$ \\
\hline & Bushnell-Rana & 37 & $09^{\circ} 25^{\prime} 12^{\prime \prime} \mathrm{N}, 76^{\circ} 21^{\prime} 06^{\prime \prime} \mathrm{W}$ & $1.8 \pm 1.5(6)$ & $1.5 \pm 1.0(6)$ \\
\hline & Bartolo & 38 & $09^{\circ} 25^{\prime} 16^{\prime \prime} \mathrm{N}, 76^{\circ} 16^{\prime} 19^{\prime \prime} \mathrm{W}$ & $9.7 \pm 2.1(3)$ & ND \\
\hline & Socorro & 39 & $09^{\circ} 24^{\prime} 21^{\prime \prime} \mathrm{N}, 76^{\circ} 12^{\prime} 01^{\prime \prime} \mathrm{W}$ & $1.0 \pm 1.1(6)$ & $0.3 \pm 0.5(6)$ \\
\hline & Bajo de los Pargos & 40 & $09^{\circ} 24^{\prime} 04^{\prime \prime} \mathrm{N}, 76^{\circ} 12^{\prime} 39^{\prime \prime} \mathrm{W}$ & ND & $0.4 \pm 0.5(5)$ \\
\hline & El Inglés & 41 & $09^{\circ} 23^{\prime} 54^{\prime \prime} \mathrm{N}, 76^{\circ} 10^{\prime} 07^{\prime \prime} \mathrm{W}$ & $0.5 \pm 0.5(6)$ & $0.8 \pm 1.0(6)$ \\
\hline & Alicia & 42 & $09^{\circ} 23^{\prime} 41^{\prime \prime} \mathrm{N}, 76^{\circ} 10^{\prime} 08^{\prime \prime} \mathrm{W}$ & $1.0 \pm 1.3(6)$ & $0.3 \pm 0.8(6)$ \\
\hline & Venados & 43 & $09^{\circ} 23^{\prime} 06^{\prime \prime} \mathrm{N}, 76^{\circ} 10^{\prime} 10^{\prime \prime} \mathrm{W}$ & $1.0 \pm 2.0(6)$ & $0.8 \pm 0.4(5)$ \\
\hline & Fondo Loco & 44 & $09^{\circ} 22^{\prime} 52^{\prime \prime} \mathrm{N}, 76^{\circ} 12^{\prime} 35^{\prime \prime} \mathrm{W}$ & ND & $0.6 \pm 0.9(5)$ \\
\hline & Planchón & 45 & $09^{\circ} 20^{\prime} 40^{\prime \prime} \mathrm{N}, 76^{\circ} 10^{\prime} 47^{\prime \prime} \mathrm{W}$ & ND & $0.6 \pm 0.5(5)$ \\
\hline \multirow{9}{*}{ Capurganá } & Cabo Tiburón/Tiburón cape & 46 & $08^{\circ} 40^{\prime} 18^{\prime \prime} \mathrm{N}, 77^{\circ} 21^{\prime} 26^{\prime \prime} \mathrm{W}$ & $1.6 \pm 2.1(5)$ & $3.2 \pm 2.2(5)$ \\
\hline & El Palmar & 47 & $08^{\circ} 39^{\prime} 51^{\prime \prime} \mathrm{N}, 77^{\circ} 21^{\prime} 16^{\prime \prime} \mathrm{W}$ & $0.2 \pm 0.4(5)$ & $1.0 \pm 0.8(5)$ \\
\hline & Piscina de Dios & 48 & $08^{\circ} 38^{\prime} 49^{\prime \prime} \mathrm{N}, 77^{\circ} 20^{\prime} 38^{\prime \prime} \mathrm{W}$ & $0.6 \pm 0.9(5)$ & ND \\
\hline & Bajo Naui & 49 & $08^{\circ} 38^{\prime} 44^{\prime \prime} \mathrm{N}, 77^{\circ} 20^{\prime} 23^{\prime \prime} \mathrm{W}$ & $1.4 \pm 2.1(5)$ & $1.4 \pm 1.1(5)$ \\
\hline & La Grieta & 50 & $08^{\circ} 38^{\prime} 30^{\prime \prime} \mathrm{N}, 77^{\circ} 20^{\prime} 11^{\prime \prime} \mathrm{W}$ & $0.2 \pm 0.4(5)$ & $0.6 \pm 1.3(5)$ \\
\hline & Bajo Carey & 51 & $08^{\circ} 38^{\prime} 29^{\prime \prime} \mathrm{N}, 77^{\circ} 20^{\prime} 04^{\prime \prime} \mathrm{W}$ & $1.0 \pm 1.0(5)$ & $1.2 \pm 0.8(5)$ \\
\hline & El Regalo & 52 & $08^{\circ} 37^{\prime} 45^{\prime \prime} \mathrm{N}, 77^{\circ} 20^{\prime} 25^{\prime \prime} \mathrm{W}$ & ND & $0.2 \pm 0.4(5)$ \\
\hline & Aguacate & 53 & $08^{\circ} 36^{\prime} 43^{\prime \prime} \mathrm{N}, 77^{\circ} 19^{\prime} 16^{\prime \prime} \mathrm{W}$ & $0.8 \pm 0.8(5)$ & $0.5 \pm 1.0(5)$ \\
\hline & Isla Pájaro/Pájaro island & 54 & $08^{\circ} 36^{\prime} 02^{\prime \prime} \mathrm{N}, 77^{\circ} 17^{\prime} 31^{\prime \prime} \mathrm{W}$ & $0.2 \pm 0.4(5)$ & $1.3 \pm 1.0(5)$ \\
\hline
\end{tabular}

peces león en 185 transectos de 48 sitios. De un total de 85 inmersiones, el invasor se observó en 75 , para una frecuencia de avistamiento de $88 \%$, lo que permite considerar la especie como frecuente en los lugares y años de muestreo (> $70 \%$ ), de acuerdo con los estándares de Schmitt y Sullivan (1996). Los sitios donde no se registró la especie durante los censos fueron Pavitos, en el archipiélago del Rosario, que se visitó en ambos años $(n=11)$, y Bajo Picuas $(n=4)$, Bajo del Medio $(n=4)$, Bajo Tiosolda $(n=4)$, isla Mangle $(n=4) y$ Bajo Minalta $2(n=4)$, que están en el archipiélago de San Bernardo y que se visitaron solamente en 2016 (Tabla 1).

Durante el estudio se registraron valores de densidad entre 0 y $12 \mathrm{ind} / 250 \mathrm{~m}^{2}$ (480 ind/ha), con un promedio general de $0,8 \pm 1,3 \mathrm{ind} / 250 \mathrm{~m}^{2}$ (32 ind/ha). En 2015, la densidad promedio fue de $0,9 \pm 1,6$, y en 2016 fue de $0,7 \pm 1,1 \mathrm{ind} / 250 \mathrm{~m}^{2}$. La densidad promedio más alta por sitio fue de $9,7 \pm 2,1 \mathrm{ind} / 250 \mathrm{~m}^{2}$ (388 ind/ha) y se registró en Bartolo (isla Fuerte) en octubre de 2015. La localidad con la densidad promedio más alta en los dos años fue isla Fuerte con $1,2 \pm 2,8 \mathrm{ind} / 250 \mathrm{~m}^{2}$ (48 ind/ha), seguido de Capurganá y Cartagena con $0,9 \pm 1,3 \mathrm{ind} / 250 \mathrm{~m}^{2}$ y $0,9 \pm 1,2 \mathrm{ind} / 250 \mathrm{~m}^{2}$, of $88 \%$, which allows considering the species as frequent in the locations and years of sampling (> $70 \%)$ according to the standards of Schmitt and Sullivan (1996). The sites where the species was not registered during the censuses were Pavitos in the Rosario archipelago, which was visited both years $(n=11)$; and Bajo Picuas $(n=4)$, Bajo del Medio $(\mathrm{n}=4)$, Bajo Tiosolda $(\mathrm{n}=4)$, Mangle island $(\mathrm{n}=4)$ and Bajo Minalta $2(n=4)$, which are in the archipelago of San Bernardo and which were visited only in 2016 (Table 1).

Density values between 0 to $12 \mathrm{ind} / 250 \mathrm{~m}^{2}$ (480 ind/ha) were recorded during the study, with a general average of $0.8 \pm 1.3 \mathrm{ind} / 250 \mathrm{~m}^{2}$ (32 ind $/$ ha). In 2015 the average density was $0.9 \pm 1.6$, and in 2016 it was $0.7 \pm 1.1 \mathrm{ind} / 250 \mathrm{~m}^{2}$. The highest average density per site was $9.7 \pm 2.1 \mathrm{ind} / 250 \mathrm{~m}^{2}$ (388 ind/ha) and was registered in Bartolo (Fuerte island) in October 2015. The locality with the highest average density in the two years was Fuerte island with $1.2 \pm 2.8 \mathrm{ind} / 250 \mathrm{~m}^{2}$ (48 ind/ha), followed by Capurganá and Cartagena with $0.9 \pm 1.3 \mathrm{ind} / 250 \mathrm{~m}^{2}$ and $0.9 \pm 1.2 \mathrm{ind} / 250 \mathrm{~m}^{2}$ respectively. When extrapolated to hectares the highest average density per site is similar to 
respectivamente. La densidad promedio más alta por sitio cuando se extrapola a hectáreas es similar a la densidad máxima de pez león reportada en Colombia por González-Corredor et al. (2016), que es de 379,03 ind/ha durante 2012 en la isla de San Andrés. Los valores de densidad del pez león de San Andrés y Bajo Bartolo están cerca de algunas de las densidades más altas reportadas para la especie en el área invadida por estudios que utilizaron métodos similares (Tabla 2).

Bartolo es una elevación del fondo marino ubicada a unos $10 \mathrm{~km}$ al noroeste de isla Fuerte, cuya parte más somera tiene alrededor de $35 \mathrm{~m}$ de profundidad. Esto hace que el sitio no sea adecuado para la pesca submarina o el buceo recreativo, y por lo tanto, que la extracción del pez león haya sido inusual o ausente en Bartolo antes de este trabajo. Otros lugares con densidades significativas de pez león fueron Bushnell-Laberinto $\left(2,8 \pm 2,5 \mathrm{ind} / 250 \mathrm{~m}^{2}\right)$, también en isla Fuerte, y cabo Tiburón, en la localidad de Capurganá $\left(3,2 \pm 2,2 \mathrm{ind} / 250 \mathrm{~m}^{2}\right)$. En términos generales, estos son sitios remotos, donde las acciones para controlar la invasión son poco frecuentes o nulas por problemas logísticos para el buceo y una presencia limitada de las autoridades ambientales debido a que no son áreas realmente protegidas. La densidad media más baja del pez león se registró en el archipiélago de San Bernardo con solo 13,7 ind/ha $\left(0,3 \pm 0,7 \mathrm{ind} / 250 \mathrm{~m}^{2}\right)$, e incluso estuvo ausente en cinco de los nueve sitios muestreados en esta localidad (Tabla 1).

Si se asume que, en los dos años, la densidad promedio en todos los sitios $\left(0,8 \pm 1,3 \mathrm{ind} / 250 \mathrm{~m}^{2} ; 32 \mathrm{ind} / \mathrm{ha}\right)$ fue representativa de la abundancia de pez león en el Caribe continental colombiano, se puede decir que este valor fue bajo en comparación con las densidades reportadas en trabajos con métodos similares (i.e. censos visuales en transectos) en otros lugares del Caribe como Bahamas, islas Caimán, Cuba, Belice, México, Costa Rica, Venezuela, y Curazao (Tabla 2). En Colombia, se han registrado densidades mucho más altas en sondeos locales, como el de González-Corredor et al. (2016) en San Andrés, y en el estudio de García-Urueña et al. (2015) en Santa Marta (Tabla 2). Sin embargo, al hacer comparaciones sobre la densidad del pez león entre estudios, se debe considerar un conjunto de factores como la profundidad, la fecha de muestreo, el tiempo transcurrido desde la llegada del invasor, el tamaño del transecto (largo y ancho) y la escala espacial del muestreo, entre otros.

Al considerar solo densidades reportadas por estudios que incluyeron múltiples ubicaciones en una escala regional (e.g. superior a $100 \mathrm{~km}$ ), encontramos valores similares a la densidad promedio registrada aquí. Por ejemplo, Agudo y the maximum density of lionfish reported in Colombia by González-Corredor et al. (2016), that is of $379.03 \mathrm{ind} / \mathrm{ha}$ during 2012 in San Andrés island. Both, San Andrés and Bajo Bartolo's lionfish density values are near to some of the highest densities reported for the species on the invaded area by studies that used similar methods (Table 2).

Bajo Bartolo is a seafloor elevation located about $10 \mathrm{~km}$ northwest of Fuerte island, which shallow part is around $35 \mathrm{~m}$ deep, making the site unsuitable for spearfishing or recreational diving so that lionfish removal has been unusual or absent before this work. Other places with significant densities of lionfish were Bushnell-Laberinto $\left(2.8 \pm 2.5 \mathrm{ind} / 250 \mathrm{~m}^{2}\right)$, also on Fuerte island, and Tiburón cape in the locality of Capurganá $\left(3.2 \pm 2.2 \mathrm{ind} / 250 \mathrm{~m}^{2}\right)$. In general terms, these are remote sites, where actions to control the invasion are infrequent or null due to logistical issues for diving and a limited presence of environmental authorities because they are not real protected areas. The lowest average density of lionfish was recorded in San Bernardo archipelago with just $13.7 \mathrm{ind} / \mathrm{ha}\left(0.3 \pm 0.7 \mathrm{ind} / 250 \mathrm{~m}^{2}\right)$, being even absent in five out of nine sampled sites at this locality (Table 1).

Assuming that the average density throughout sites in the two years $\left(0.8 \pm 1.3 \mathrm{ind} / 250 \mathrm{~m}^{2}\right)(32 \mathrm{ind} / \mathrm{ha})$ was representative of lionfish abundance on the Colombian continental Caribbean, then we can say that this value was low compared to densities reported in works with similar methods (i.e. UVC in transects) in other places in the Caribbean, such as in the Bahamas, Cayman islands, Cuba, Belize, Mexico, Costa Rica, Venezuela, and Curacao (Table 2). In Colombia, much higher densities have been recorded in local surveys, like that by González-Corredor et al. (2016) in San Andrés, and the study by García-Urueña et al. (2015) in Santa Marta (Table 2). However, when making comparisons on lionfish density among studies, a suite of factors must be considered, such as depth, date of sampling, the time elapsed since the arrival of the invader, transect size (length and width), and spatial scale of the survey, among others.

When considering only densities reported by studies including multiple locations at a regional scale (e.g. greater than $100 \mathrm{~km}$ ), we found similar values to the average density reported here. For example, Agudo and Klein (2014) sampled five localities distributed over more than $400 \mathrm{~km}$ of the Venezuelan coast in 2013 and reported $25.83 \mathrm{ind} / \mathrm{ha}$; Whitfield et al. (2007) scan sites along $160 \mathrm{~km}$ off the North Carolina coast in 2004 and 
Tabla 2. Densidades de pez león estimadas mediante censos visuales en estudios realizados en varios lugares del Atlántico occidental. DE: Desviación estándar; n: número de transectos.

\begin{tabular}{|c|c|c|c|c|c|}
\hline Sitio/Site & $\begin{array}{l}\text { Primer } \\
\text { registro/ } \\
\text { First } \\
\text { recording }\end{array}$ & $\begin{array}{l}\text { Fecha del muestreo/ } \\
\text { Date of sampling }\end{array}$ & $\begin{array}{l}\text { Tamaño } \\
\text { del transecto/ } \\
\text { Transect size }\end{array}$ & $\begin{array}{l}\text { Densidad media } \pm \text { DE, } \mathbf{n} \\
\text { (profundidad)/Average } \\
\text { density } \pm \text { SD, } \mathbf{n} \\
\text { (depth) }\end{array}$ & Referencia/Reference \\
\hline $\begin{array}{l}\text { Parque Tayrona, Cartagena, archipiélago } \\
\text { del Rosario, archipiélago de San } \\
\text { Bernardo, isla Fuerte y Capurganá, } \\
\text { Colombia/ } \\
\text { Tayrona Park, Cartagena, Rosario } \\
\text { archipelago, San Bernardo archipelago, } \\
\text { Fuerte Island and Capurganá, Colombia }\end{array}$ & 2009 & $\begin{array}{l}\text { Ago-2015 a sep-2016/ } \\
\text { Aug-2015 to Sep-2016 }\end{array}$ & $25 \times 10 \mathrm{~m}$ & $\begin{array}{l}0.8 \pm 1.3 \mathrm{ind} / 250 \mathrm{~m}^{2} \\
\mathrm{n}=452(4.5-44 \mathrm{~m})\end{array}$ & $\begin{array}{l}\text { Este estudio/ } \\
\text { Present study }\end{array}$ \\
\hline San Andrés, Colombia & 2008 & $\begin{array}{l}\text { Ago-2011 a mar-2013/ } \\
\text { Aug-2011 to Mar-2013 }\end{array}$ & $\begin{array}{l}100 \times 3 \mathrm{~m} \\
200 \times 3 \mathrm{~m}\end{array}$ & $\begin{array}{l}379.03 \pm 220 \mathrm{ind} / \mathrm{ha} \\
(10-22 \mathrm{~m})\end{array}$ & $\begin{array}{l}\text { González-Corredor } \\
\text { et al. }(2016)\end{array}$ \\
\hline $\begin{array}{l}\text { Santa Marta y Parque Tayrona, Colombia/ } \\
\text { Santa Marta and Tayrona Park, Colombia }\end{array}$ & 2009 & $\begin{array}{l}\text { Ene-dic 2012/ } \\
\text { Jan-Dec } 2012\end{array}$ & $30 \times 4 \mathrm{~m}$ & $\begin{array}{l}2.6 \pm 2.1 \mathrm{ind} / 120 \mathrm{~m}^{2} \\
(10-12 \mathrm{~m})\end{array}$ & $\begin{array}{l}\text { García-Urueña et al. } \\
\qquad(2015)\end{array}$ \\
\hline $\begin{array}{l}\text { Santa Marta y Parque Tayrona, Colombia/ } \\
\text { Santa Marta and Tayrona Park, Colombia }\end{array}$ & 2009 & $\begin{array}{l}\text { Dic-2011 a dic-2012/ } \\
\text { Dec-2011 to Dec-2012 }\end{array}$ & $30 \times 10 \mathrm{~m}$ & $\begin{aligned} & 2.60 \pm 2.12 \mathrm{ind} / 300 \mathrm{~m}^{2} \\
&(10-12 \mathrm{~m})\end{aligned}$ & $\begin{array}{l}\text { Coronado-Carrascal } \\
\text { et al. }(2015)\end{array}$ \\
\hline $\begin{array}{l}\text { Parque Tayrona, Colombia/ } \\
\text { Tayrona Park, Colombia }\end{array}$ & 2009 & 2012 & $50 \times 5 \mathrm{~m}$ & $\begin{array}{l}2.9 \pm 0.9 \mathrm{ind} / \mathrm{ha} \\
\mathrm{n}=288(9-11 \mathrm{~m})\end{array}$ & $\begin{array}{l}\text { Bayraktarov et al. } \\
\qquad(2014)\end{array}$ \\
\hline Dos Mosquises, Venezuela & 2010 & Mar-2011, mar-2013 & $30 \times 2 \mathrm{~m}$ & $\begin{array}{l}121 \pm 164 \mathrm{ind} / \mathrm{ha} \\
\mathrm{n}=22(6-12 \mathrm{~m})\end{array}$ & Elise et al. (2015) \\
\hline $\begin{array}{l}\text { Parque Nacional Morrocoy, Ocumare } \\
\text { de la Costa, Chichirivichi de la Costa, } \\
\text { Chuspa y Parque Nacional Mochina, } \\
\text { Venezuela/ } \\
\text { Morrocoy National Park, Ocumare de la } \\
\text { Costa, Chichirivichi de la Costa, Chuspa } \\
\text { and Mochina National Park, Venezuela }\end{array}$ & 2009 & 2013 & $30 \times 4 \mathrm{~m}$ & $\begin{array}{c}25.83 \pm 66.51 \mathrm{ind} / \mathrm{ha} \\
\mathrm{n}=200(5-20 \mathrm{~m})\end{array}$ & $\begin{array}{l}\text { Agudo and Klein } \\
\qquad(2014)\end{array}$ \\
\hline $\begin{array}{l}\text { Bonaire, Caribe sur/ } \\
\text { Bonaire, southern Caribbean }\end{array}$ & 2009 & $\begin{array}{l}\text { Jun-ago 2011/ } \\
\text { Jun-Aug 2011 }\end{array}$ & $50 \times 40 \mathrm{~m}$ & $\begin{array}{c}66 \text { ind } / \text { ha, } \\
\mathrm{n}=151(5,25,35 \mathrm{~m})\end{array}$ & De León et al. (2013) \\
\hline $\begin{array}{l}\text { Curazao, Caribe sur/ } \\
\text { Curacao, southern Caribbean }\end{array}$ & 2009 & $\begin{array}{l}\text { Jun-ago 2011/ } \\
\text { Jun-Aug 2011 }\end{array}$ & $50 \times 40 \mathrm{~m}$ & $\begin{array}{c}127 \mathrm{ind} / \mathrm{ha}, \\
\mathrm{n}=147(5,25,35 \mathrm{~m})\end{array}$ & De León et al. (2013) \\
\hline $\begin{array}{l}\text { Costa Caribe sur de Costa Rica/ } \\
\text { Southern Caribbean coast of Costa Rica }\end{array}$ & 2008 & Mar-jun 2011 & $15-43 \mathrm{~m} \times 5 \mathrm{~m}$ & $\begin{array}{c}92 \pm 130 \mathrm{ind} / \mathrm{ha} \\
\mathrm{n}=26(0-7 \mathrm{~m})\end{array}$ & Sandel et al. (2015) \\
\hline $\begin{array}{l}\text { Reserva de la Biosfera Banco Chinchorro } \\
\text { (BCBR), México/ } \\
\text { Banco Chinchorro Biosphere Reserve } \\
\text { (BCBR), Mexico }\end{array}$ & 2008 & Mar-may 2013 & $30 \times 4 \mathrm{~m}$ & $\begin{array}{c}97.58 \pm 140.25 \mathrm{ind} / \mathrm{ha} \\
\mathrm{n}=130\end{array}$ & $\begin{array}{c}\text { Sabido-Itzá y } \\
\text { García-Rivas (2019) }\end{array}$ \\
\hline $\begin{array}{l}\text { Barrera Mesoamericana Belice-México/ } \\
\text { Mesoamerican Barrier Belize-Mexico }\end{array}$ & 2008 & May-jul 2012 & $50 \times 10 \mathrm{~m}$ & $1.6 \mathrm{ind} / 100 \mathrm{~m}^{2}$ & Hackerott et al. (2013) \\
\hline Little Cayman Island, UK & 2008 & 2011 & $50 \times 4 \mathrm{~m}$ & $233-650 \mathrm{ind} / \mathrm{ha}$ & Frazer et al. (2012) \\
\hline $\begin{array}{l}\text { Parque Marino Cayos de San Felipe, } \\
\text { Cuba/ } \\
\text { Cayos de San Felipe Marine Park, Cuba }\end{array}$ & 2009 & $2013-2015$ & $50 \times 2 \mathrm{~m}$ & $\begin{array}{c}0.4 \text { ind } / 100 \mathrm{~m}^{2} \\
\mathrm{n}=72(15 \mathrm{~m}) \\
0.3 \mathrm{ind} / 100 \mathrm{~m}^{2}, \mathrm{n}=72(25 \mathrm{~m})\end{array}$ & $\begin{array}{l}\text { De la Guardia et al. } \\
\qquad(2017)\end{array}$ \\
\hline $\begin{array}{l}\text { Parque Nacional Guanahacabibes, Cuba/ } \\
\text { Guanahacabibes National Park, Cuba }\end{array}$ & 2009 & $\begin{array}{c}\text { Dic-2010, sep-2011, } \\
\text { abr-2012, jun-2013, } \\
\text { feb-2014/ } \\
\text { Dec-2010, Sep-2011, } \\
\text { Apr-2012, Jun-2013, } \\
\text { Feb-2014 }\end{array}$ & $50 \times 2 \mathrm{~m}$ & $0.031 \mathrm{ind} / \mathrm{m}^{2},(15-25 \mathrm{~m})$ & Cobián et al. (2016) \\
\hline $\begin{array}{l}\text { Arrecifes de la costa oeste de La Habana, } \\
\text { Cuba/ } \\
\text { Reefs off the west coast of La Habana, } \\
\text { Cuba }\end{array}$ & 2007 & $\begin{array}{l}\text { Jun-jul } 2012, \text { dic-2012 } \\
\text { a ene-2013/ } \\
\text { Jun-Jul 2012, Dec-2012 } \\
\text { to Jan-2013 }\end{array}$ & $50 \times 2 \mathrm{~m}$ & $\begin{aligned} 1.6 & \pm 1.8 \mathrm{ind} / 100 \mathrm{~m}^{2} \\
\mathrm{n} & =12(10-12 \mathrm{~m})\end{aligned}$ & $\begin{array}{l}\text { García-Rodríguez et al. } \\
\text { (2015) }\end{array}$ \\
\hline
\end{tabular}

Table 2. Lionfish densities estimated through visual censuses in studies carried out in several places of the Western Atlantic. SD: Standard deviation; n: number of transects. 


\begin{tabular}{|c|c|c|c|c|c|}
\hline Sitio/Site & $\begin{array}{l}\text { Primer } \\
\text { registro/ } \\
\text { First } \\
\text { recording }\end{array}$ & $\begin{array}{l}\text { Fecha del muestreo/ } \\
\text { Date of sampling }\end{array}$ & $\begin{array}{c}\text { Tamaño } \\
\text { del transecto/ } \\
\text { Transect size }\end{array}$ & $\begin{array}{c}\text { Densidad media } \pm \text { DE, } \mathrm{n} \\
\text { (profundidad)/Average } \\
\text { density } \pm \text { SD, }, \\
(\text { depth) }\end{array}$ & Referencia/Reference \\
\hline Jardines de la Reina, Cuba & 2007 & May-jun 2011 & $50 \times 10 \mathrm{~m}$ & $1.5 \mathrm{ind} / 100 \mathrm{~m}^{2}$ & Hackerott et al. (2013) \\
\hline Eleuthera, Bahamas & 2005 & 2009 & $50 \times 10 \mathrm{~m}$ & $4.4 \pm 0.5 \mathrm{ind} / 100 \mathrm{~m}^{2}, \mathrm{n}=12$ & Hackerott et al. (2013) \\
\hline Eleuthera, Bahamas & 2007 & $\begin{array}{l}\text { Jun-2011 a feb-2012/ } \\
\text { Jun-2011 to Feb-2012 }\end{array}$ & $24-30 \times 4 \mathrm{~m}$ & $3 \pm 6$ ind $/ 100 \mathrm{~m}^{2}, \mathrm{n}=33$ & Green et al. (2013) \\
\hline New Providence, Bahamas & 2004 & May-jul 2008 & $50 \times 10 \mathrm{~m}$ & $\begin{array}{l}393.3 \pm 144.4 \mathrm{ind} / \mathrm{ha} \\
\mathrm{n}=12(12-20 \mathrm{~m})\end{array}$ & Green and Côté (2009) \\
\hline New Providence, Bahamas & 2004 & May-jul 2008 & $50 \times 10 \mathrm{~m}$ & $\begin{array}{l}101.7 \pm 103.0 \mathrm{ind} / \mathrm{ha} \\
\mathrm{n}=75(5-20 \mathrm{~m})\end{array}$ & Darling et al. (2011) \\
\hline $\begin{array}{l}\text { Carolina del Norte, EE. UU./ } \\
\text { North Carolina, USA }\end{array}$ & 2000 & $\begin{array}{l}\text { Ago-2004/ } \\
\text { Aug-2004 }\end{array}$ & $50 \mathrm{~m}, 100 \mathrm{~m}$ & $\begin{array}{l}21.2 \pm 5.1 \mathrm{ind} / \mathrm{ha} \\
\mathrm{n}=17(30-45 \mathrm{~m})\end{array}$ & Whitfield et al. 2007 \\
\hline
\end{tabular}

Klein (2014) muestrearon cinco localidades distribuidas en más de $400 \mathrm{~km}$ de costa venezolana en 2013 e informaron 25,83 ind/ha; Whitfield et al. (2007) exploraron sitios a lo largo de $160 \mathrm{~km}$ en la costa de Carolina del Norte en 2004 y reportaron densidades de 21,2 ind/ha. Por lo tanto, parece que las densidades de pez león obtenidas por estudios con varias localidades dispersas en grandes áreas pueden ser más diagnósticas de esta invasión biológica a escala nacional. Además, tales estudios permiten hacer comparaciones directas entre lugares por la consistencia en los métodos de muestreo, lo que destaca la importancia de establecer programas de monitoreo estandarizados en el país.

En Colombia no existen estudios que evalúen la abundancia de pez león en una escala espacial amplia mediante censos visuales en transectos. Recientemente, RojasVélez et al. (2019), evaluando una hipótesis de resistencia biológica, contaron $P$. volitans mediante censos visuales errantes y reportaron densidades equivalentes a $84 \mathrm{ind} / \mathrm{ha}$ $\left(0,84 \pm 0,89 \mathrm{ind} / 100 \mathrm{~m}^{2}\right)$. Las diferencias en los métodos, la intensidad de muestreo y los sitios impiden una comparación directa con los resultados de este estudio; sin embargo, a pesar del menor esfuerzo de muestreo (44 censos en 24 sitios versus 452 transectos en 54 sitios), su densidad promedio es tres veces mayor que la registrada en esta investigación, lo que podría explicarse por diferencias metodológicas y sus conteos en la isla de San Andrés, donde se han registrado las densidades más altas de pez león en Colombia (GonzálezCorredor et al., 2016).

El único lugar en Colombia con varias publicaciones sobre la abundancia del pez león estimada a través de censos visuales en transectos es el PNN Tayrona, y la variabilidad en los valores de densidad es notoria, pues van desde 2,9 ind/ha (Bayraktarov et al., 2014) hasta 216,7 ind/ha (García-Urueña reported densities of $21.2 \mathrm{ind} / \mathrm{ha}$. Therefore, it seems like lionfish densities obtained by studies with several localities scattered in large areas can be more diagnostic of this biological invasion on a national scale. Furthermore, such studies allow direct comparisons to be made among places by the consistency in sampling methods, highlighting the importance of establishing standardized monitoring programs in the country.

Studies on a broad spatial scale evaluating lionfish abundance through underwater visual censuses in transects do not exist in Colombia. Recently, RojasVélez et al. (2019) evaluating a biological resistance hypothesis counted $P$. volitans through roving visual censuses and reported densities equivalent to $84 \mathrm{ind} / \mathrm{ha}$ $\left(0.84 \pm 0.89 \mathrm{ind} / 100 \mathrm{~m}^{2}\right)$. Differences in methods, sampling intensity, and sites preclude a direct comparison with our results; however, despite lower sampling effort (44 censuses in 24 sites versus 452 transects in 54 sites), their average density is three-fold than the one herein reported, which could be explained by methodological differences and their counts in San Andrés island, where highest lionfish densities in Colombia have been recorded (González-Corredor et al., 2016).

The only one location in Colombia with several publications on lionfish abundance estimated through visual censuses in transects is Tayrona NNP, and the variability in density values are remarkable, ranging from $2.9 \mathrm{ind} / \mathrm{ha}$ (Bayraktarov et al., 2014) up to $216.7 \mathrm{ind} / \mathrm{ha}$ (García-Urueña et al., 2015). The estimated abundances in this study for that locality (Table 1) are more similar to those in Coronado-Carrascal et al. (2015), who reported values equivalent to $68,63.7$, and $37 \mathrm{ind} / \mathrm{ha}$ for Cinto, Chengue, and Aguja island. It cannot be ruled out that 
et al., 2015). Las abundancias estimadas en este estudio para esa localidad (Tabla 1) son más similares a las de Coronado-Carrascal et al. (2015), que reportaron valores equivalentes a 68, 63,7 y $37 \mathrm{ind} / \mathrm{ha}$ para Cinto, Chengue e isla Aguja. No se puede descartar que toda esta variabilidad en las estimaciones de abundancia del pez león, incluso en un solo lugar, puede ser un reflejo de poblaciones altamente dinámicas por las variaciones en el esfuerzo reproductivo y el éxito del reclutamiento versus la mortalidad natural más la mortalidad por pesca debido a esfuerzos de control diferentes en cada localidad. Sin embargo, en ausencia de resultados de un monitoreo a largo plazo con métodos estándar, es más fácil atribuir esa variabilidad a las diferencias en los métodos y al esfuerzo de muestreo entre los diferentes estudios. De ahí la importancia de establecer un programa nacional de monitoreo para la invasión por pez león.

Las densidades de pez león en la mayoría de los sitios estudiados fueron similares entre años; sin embargo, se obtuvieron algunos valores extremos con algunos sitios que muestran densidades mucho más altas que el promedio, y otros donde no se registró la presencia de pez león (Figura 2). La prueba de la mediana de Mood mostró que no hubo diferencias significativas en las densidades de pez león entre los años muestreados $\left(X_{1}^{2}=1,70 ; p=0,19\right)$, ni entre las localidades $\left(X_{5}^{2}=5,93 ; p=0,31\right)$, ni entre las profundidades de muestreo $\left(X_{3}^{2}=1,47 ; p=0,68\right)$.

Las variaciones en la densidad del pez león entre localidades podrían deberse a diferencias en el régimen de acciones de control, principalmente porque los torneos de pez león y la cacería frecuente que hacen las escuelas de buceo están ocurriendo solo en algunas áreas. Por ejemplo, las localidades Rosario, San Bernardo y Tayrona, al ser áreas protegidas en el Sistema Nacional de Parques Naturales, tienen acciones de control de la invasión desde 2010 (e.g. Resolución MAVDT 0132 de 2010). Además, debido a la proximidad de las ciudades turísticas a estas áreas, la cacería del pez león por parte de pescadores y buzos es frecuente (L. Chasqui obs. pers.). Por el contrario, Capurganá e isla Fuerte son lugares remotos que carecen de figuras de protección y donde la presencia de buzos es escasa; por lo tanto, las acciones de control de las poblaciones del invasor han sido prácticamente inexistentes. Diferentes estudios en el AO destacan la necesidad de controlar el número de peces león mediante la cacería constante y los torneos de pesca, que son quizás las únicas acciones disponibles hasta la fecha para enfrentar la invasión biológica por $P$. volitans (Barbour et al., 2011; Frazer et al., 2012; De León et al., 2103; Green et al., 2017). all this variability in lionfish abundance estimates, even in one location, can be a reflection of highly dynamic populations due to variations in reproductive effort and recruitment success versus natural mortality, plus fishing mortality caused by differential control efforts in each locality. However, in the absence of results from a longterm monitoring with standard methods, it is easier to attribute that variability to differences in methods and sampling effort among the different studies. Hence the importance to establish a national monitoring program for lionfish invasion.

Lionfish densities at the majority of surveyed sites were similar between years; however, some extreme values were present, with a few sites showing densities much higher than average and others where the presence of lionfish was not recorded (Figure 2). The Mood median test showed that there were no significant differences in lionfish densities between the sampled years $\left(X_{1}^{2}=1.70\right.$; $\mathrm{p}=0.19)$, neither among localities $\left(\mathrm{X}_{5}^{2}=5.93 ; \mathrm{p}=0.31\right)$ and nor between sampling depths $\left(\mathrm{X}_{3}^{2}=1.47 ; \mathrm{p}=0.68\right)$.

Variations in lionfish density between localities could be due to differences in the regime of control actions, mainly because lionfish derbies and frequent culling by diving schools are happening just in some areas. For example, the localities Rosario, San Bernardo, and Tayrona, are protected areas into the National System of Natural Parks and have control actions of the invasion since 2010 (e.g. Resolution MAVDT 0132 of 2010). Furthermore, due to the proximity of touristic cities to these areas, lionfish culling by fishers and divers are frequent (L. Chasqui pers. obs.). On the contrary, Capurganá and Fuerte island are remote places without protection status and where the presence of divers is scarce; therefore, control actions of the invader populations have been practically nonexistent. Different studies in the WA stand out the need of controlling lionfish numbers through constant culling and derbies, which are maybe the only actions available until date to face the biological invasion by $P$. volitans (Barbour et al., 2011; Frazer et al., 2012; De León et al., 2103; Green et al., 2017).

Variations in lionfish abundance (and size) have been previously documented in different locations on the invaded area, often increasing with depth (e.g. Claydon et al., 2012; González et al., 2016; Hunt et al., 2019). Among the explanations, a highest fishing pressure in shallow waters has been mentioned. However, fishing pressure decreases with depth until being virtually non-existent 


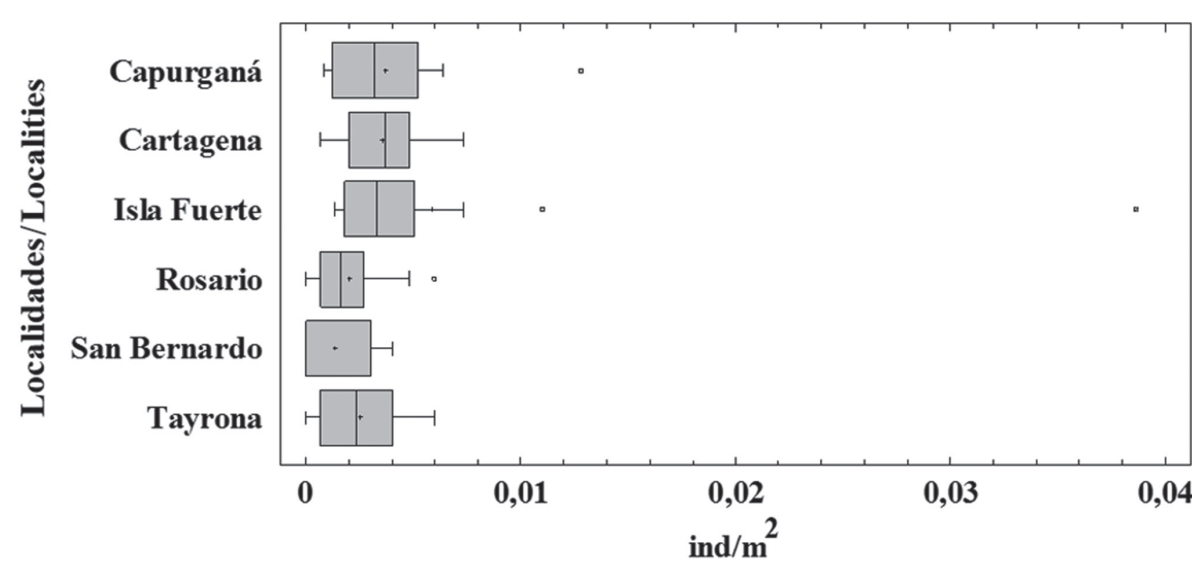

a)

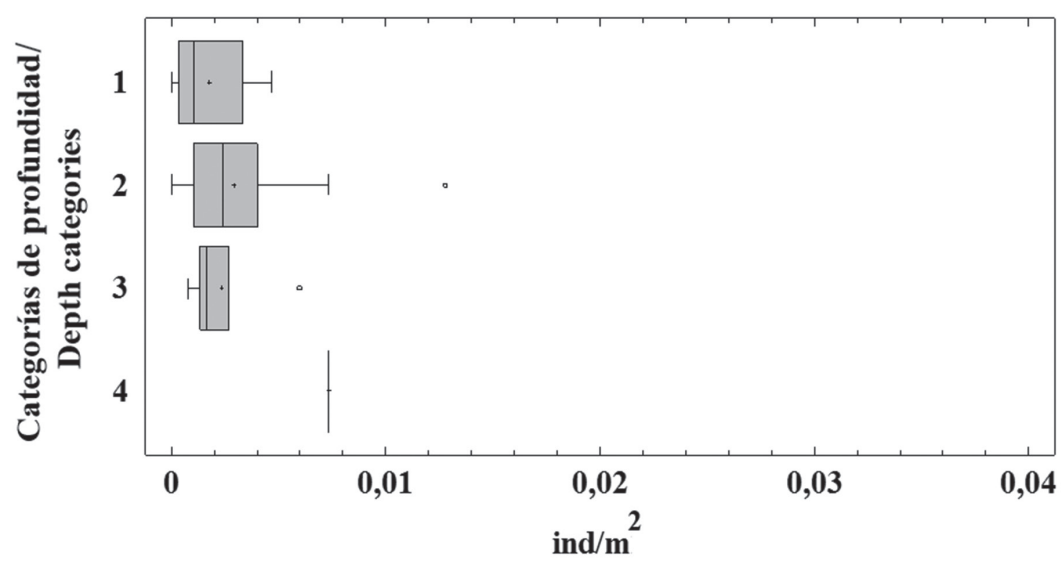

b)

Figura 2. a) Densidad del pez león en seis localidades de la costa Caribe colombiana: Capurganá $(n=77)$, Cartagena $(n=63)$, isla Fuerte $(n=84)$, Rosario ( $\mathrm{n}=99)$, San Bernardo $(\mathrm{n}=38)$, Tayrona $(\mathrm{n}=88)$. b) Densidad del pez león a diferentes profundidades: $1(1-10 \mathrm{~m}, \mathrm{n}=25), 2(>10-20 \mathrm{~m}$, $\mathrm{n}=108), 3(>20-30 \mathrm{~m}, \mathrm{n}=34), 4(>30-40 \mathrm{~m}, \mathrm{n}=3)$. La línea dentro de las cajas representa la mediana, los límites de las cajas son los percentiles 25 y 75 , los bigotes marcan los valores mínimo y máximo, y los puntos por fuera de las cajas son datos atípicos.
Figure 2. a) Lionfish density in six localities from the Colombian Caribbean coast: Capurganá $(n=77)$, Cartagena $(n=63)$, Fuerte island $(\mathrm{n}=84)$, Rosario $(\mathrm{n}=99)$, San Bernardo $(\mathrm{n}=38)$, Tayrona $(\mathrm{n}=88) . \mathrm{b})$ Lionfish density at different depths: $1(1-10 \mathrm{~m}, \mathrm{n}=25), 2(>10-20 \mathrm{~m}$, $\mathrm{n}=108), 3(>20-30 \mathrm{~m}, \mathrm{n}=34), 4(>30-40 \mathrm{~m}, \mathrm{n}=3)$. The line inside boxes represents the median, the limits of the boxes are the $25^{\text {th }}$ and $75^{\text {th }}$ percentiles, the whiskers mark minimum and maximum values, and points outside the boxes are outliers.
Las variaciones en la abundancia (y tamaño) del pez león han sido documentadas previamente en diferentes lugares del área invadida, y a menudo aumentan con la profundidad (e.g. Claydon et al., 2012; González et al., 2016; Hunt et al., 2019). Entre las explicaciones, se ha mencionado la mayor presión de pesca en aguas poco profundas, ya que la presión de la pesca disminuye con la profundidad hasta ser prácticamente inexistente en entornos fuera del alcance del buceo recreativo (e.g. ecosistemas coralinos mesofóticos - MCE). Al respecto, Andradi-Brown et al. (2017), al estudiar poblaciones de pez león en un gradiente de profundidad, encontraron altas densidades a más de $30 \mathrm{~m}$, así como tamaños más grandes y hembras más fértiles, lo que puede estar minando los esfuerzos de control en aguas someras. Ellos señalan la necesidad de entender y in environments outside the reach of recreational diving (e.g. mesophotic coral ecosystems - MCE). In this regard, Andradi-Brown et al. (2017) studying lionfish populations in a depth gradient found high densities over $30 \mathrm{~m}$ depth, as well as larger sizes and more fertile females, which may be undermining control efforts in shallow waters. They point out the need to understand and consider the importance of MCE in the life cycle of the species, and to seek strategies to extend control actions of lionfish invasion to those environments.

Multiple factors, acting alone or synergistically, can influence the distribution and abundance of lionfish, and can be determinant on the observed differences in density among sampling sites; for example, the structural 
considerar la importancia de los MCE en el ciclo de vida de la especie, y de buscar estrategias para extender las acciones de control de la invasión del pez león a esos ambientes.

Múltiples factores que actúan solos o en sinergia pueden influir en la distribución y abundancia del pez león, y podrían haber determinado las diferencias observadas en la densidad entre los sitios de muestreo; por ejemplo, la complejidad estructural del fondo, la disponibilidad de refugios, la conectividad espacial de los parches de hábitat y la abundancia de presas, entre otros (Biggs y Olden, 2011; Hernández-Abello et al., 2015; Rivera-Aponte et al., 2019; Díaz-Ferguson y Hunter, 2019).

\section{AGRADECIMIENTOS}

El trabajo se realizó como parte del convenio 275 de 2015 entre MinAmbiente e INVEMAR, y la Resolución 0478 de MinAmbiente de 2016. Gracias a Arnold Fonseca, Maria Parrado, Javier Torres, Adolfo Sanjuan, Diana Vergara, Carlos Aponte, Arnold Llorente, Deibis Seguro y Diego Arnedo por su ayuda en la toma de datos. Gracias al personal del PNN Corales del Rosario y San Bernardo y del PNN Tayrona por el apoyo logístico durante el trabajo de campo en sus áreas protegidas. Gracias a David Forero por elaborar el mapa del área de estudio. Gracias a los evaluadores por su contribución a mejorar considerablemente el manuscrito. Número de contribución de INVEMAR \# 1266. complexity of the bottom, availability of shelters, the spatial connectivity of habitat patches, and prey abundance, among others (Biggs and Olden, 2011; Hernández-Abello et al., 2015; Rivera-Aponte et al., 2019; Díaz-Ferguson and Hunter, 2019).

\section{ACKNOWLEDGMENTS}

This work was done as part of the Agreement 275 of 2015 between MinAmbiente and INVEMAR, and the MinAmbiente Resolution 0478 of 2016. Thanks to Arnold Fonseca, Maria Parrado, Javier Torres, Adolfo Sanjuan, Diana Vergara, Carlos Aponte, Arnold Llorente, Deibis Seguro and Diego Arnedo for help in data collection. Thanks to the staff of Corales del Rosario and San Bernardo NNP and Tayrona NNP for logistical support during fieldwork in their protected areas. Thanks to David Forero for preparing the map of the study area. Thanks to the anonymous reviewers for their contribution to significantly improving the manuscript. INVEMAR contribution number 1266 .

\section{BIBLIOGRAFÍA/LITERATURE CITED}

Acero P., A., D. Bustos-Montes, P. Pabón Q., C.J. Polo-Silva and A. Sanjuan M. 2019. Feeding habits of Pterois volitans: a real threat to Caribbean coral reef biodiversity. 269-314. En: Makowski, C. and C.W. Finkl (Eds). Impacts of Invasive Species on Coastal Environments. Coastal Research Library, vol. 29. Springer, Cham. $482 \mathrm{p}$

Agudo, E.A. and E. Klein. 2014. Lionfish abundance, size structure and spatial distribution along the Venezuelan coast (Pterois volitans, Pteroinae: Scorpaenidae). Rev. Biol. Trop., 62(3): 151-158.

Albins, M.A. and M.A. Hixon. 2008. Invasive Indo-Pacific lionfish Pterois volitans reduce recruitment of Atlantic coral-reef fishes. Mar. Ecol. Prog. Ser., 367: 233- 238. doi: 10.3354/meps07620

Andradi-Brown, D.A., R. Grey, A. Hendrix, D. Hitchner, C.L. Hunt, E. Gress, K. Madej, R.L. Parry, C. Régnier-McKellar, O.P. Jones, M. Arteaga, A.P. Izaguirre, A.D. Rogers and D.A. Exton. 2017. Depth-dependent effects of culling - do mesophotic lionfish populations undermine current management? R. Soc. open sci., 4: 170027. http://dx.doi.org/10.1098/rsos.170027

Ballew, N.G., N.M. Bacheler, G.T. Kellison and A.M. Schueller. 2016. Invasive lionfish reduce native abundance on a regional scale. Sci. Rep. 6, 32169; doi: 10.1038/srep32169 (2016).

Barbour, A.B., M.S. Allen, T.K. Frazer and K.D. Sherman. 2011. Evaluating the potential efficacy of invasive lionfish (Pterois volitans) removals. PLoS ONE, 6(5): e19666. doi:10.1371/journal.pone.0019666.

Bayraktarov, E., J. Alarcón-Moscoso, A. Polanco F. and C. Wild. 2014. Spatial and temporal distribution of the invasive lionfish Pterois volitans in coral reefs of Tayrona National Natural Park, Colombian Caribbean. PeerJ 2:e397; DOI 10.7717/peerj.397

Betancur-R., R., A. Hines, A. Acero P., G. Ortí, A.E. Wilbur and D.W. Freshwater. 2011. Reconstructing the lionfish invasion: insights into Greater Caribbean biogeography. J. Biogeogr., 38(7): 1281-1293. doi:10.1111/j.1365-2699.2011.02496.x 
Biggs, C.R. and J.D. Olden. 2011. Multi-scale habitat occupancy of invasive lionfish (Pterois volitans) in coral reef environments of Roatan, Honduras. Aquat. Invasions, 6(3): 347-353. doi: 10.3391/ai.2011.6.3.11

Claydon, J.A.B., M.C. Calosso and S.B. Traiger. 2012. Progression of invasive lionfish in seagrass, mangrove and reef habitats. Mar. Ecol. Prog. Ser., 448: 119-129. doi: 10.3354/meps09534

Cobián Rojas, D., P. Chevalier Monteagudo, J.J. Schmitter-Soto, R.I. Corrada Wong, H. Salvat Torres, E. Cabrera Sansón, A. García Rodríguez, A. Fernández Osorio, L. Espinosa Pantoja, D. Cabrera Guerra, L.M. Pantoja Echevaria, H. Caballero Aragón and S. Perera Valderrama. 2016. Density, size, biomass, and diet of lionfish in Guanahacabibes National Park, western Cuba. Aquat. Biol., 24: 219-226. doi: 10.3354/ab00651

Coronado-Carrascal, V., R. García-Urueña y A. Acero P. 2015. Comunidad de peces arrecifales en relación con la invasión del pez león: el caso del Caribe sur. Bol. Invest. Mar. Cost., 44(2): 291-302.

Côté, I.M., S.J. Green and M.A. Hixon. 2013. Predatory fish invaders: Insights from Indo-Pacific lionfish in the Western Atlantic and Caribbean. Biol. Conserv., 164: 50-61. http://dx.doi.org/10.1016/j.biocon.2013.04.014

Cure, K., J.L. Mcllwain and M.A. Hixon. 2014. Habitat plasticity in native Pacific red lionfish Pterois volitans facilitates successful invasion of the Atlantic. Mar. Ecol. Prog. Ser., 506: 243-253. doi: 10.3354/meps10789

Darling, E.S., S.J. Green, J.K. O’Leary and I.M. Côté. 2011. Indo-Pacific lionfish are larger and more abundant on invaded reefs: a comparison of Kenyan and Bahamian lionfish populations. Biol. Invasions, 13: 2045-2051. DOI 10.1007/s10530-011-0020-0

De la Guardia, E., D. Cobián-Rojas, L. Espinosa, Z. Hernández, L. García y J.E. Arias-González. 2017. Distribución y abundancia del pez león Pterois volitans (Scorpaeniformes: Scorpaenidae) y especies nativas asociadas en el Parque Marinos Cayos de San Felipe, Cuba. Rev. Biol. Trop., 65(1): 117-125.

De León, R., K. Vane, P. Bertuol, V.C. Chamberland, F. Simal, E. Imms and M.J.A. Vermeij. 2013. Effectiveness of lionfish removal efforts in the southern Caribbean. Endang. Species Res., 22: 175-183. doi: 10.3354/esr00542

Díaz-Ferguson, E.E. and M.E. Hunter. 2019. Life history, genetics, range expansion and new frontiers of the lionfish (Pterois volitans, Perciformes: Pteroidae) in Latin America. Reg. Stud. Mar. Sci., 31: 100793. https://doi.org/10.1016/j.rsma.2019.100793

Elise, S., I. Urbina-Barreto, H. Boadas-Gil, M. Galindo-Vivas and M. Kulbicki. 2015. No detectable effect of lionfish (Pterois volitans and P. miles) invasion on a healthy reef fish assemblage in Archipelago Los Roques National Park, Venezuela. Mar. Biol., 162: 319-330. https://doi.org/10.1007/s00227-014-2571-y

Ferreira, C.E.L., O.J. Luiz, S.R. Floeter, M.B. Lucena, M.C. Barbosa, C.R. Rocha and L.A. Rocha. 2015. First record of invasive lionfish (Pterois volitans) for the Brazilian coast. PLoS ONE 10(4): e0123002. doi:10.1371/journal.pone.0123002

Fogg, A.Q., N.J. Brown-Peterson and M.S. Peterson. 2017. Reproductive life history characteristics of invasive red lionfish (Pterois volitans) in the northern Gulf of Mexico. Bull. Mar. Sci., 93(3): 791-813. https://doi.org/10.5343/bms.2016.1095

Frazer, T.K., C.A. Jacoby, M.A. Edwards, S.C. Barry and C.M. Manfrino. 2012. Coping with the lionfish invasion: can targeted removals yield beneficial effects? Rev. Fish. Sci., 20(4): 185-191, DOI: 10.1080/10641262.2012.700655

García-Rodríguez, A., P.P. Chevalier M., E. Cabrera S., H. Caballero A., J.L. Hernández L. 2015. Densidad y biomasa de Pterois volitans/miles (Teleostei: Scorpaenidae) en arrecifes del litoral oeste de la Habana, Cuba. Rev. Invest. Mar., 35(1): 21-36.

García-Urueña, R., A. Acero P. y V. Coronado-Carrascal. 2015. Comunidad de peces arrecifales en presencia del pez león (Pterois volitans) en Santa Marta, Caribe colombiano. Rev. MVZ Córdoba, 20 (Supl.): 4989-5003.

Gardner, P.G., T.K. Frazer, C.A. Jacoby and R.P.E. Yanong. 2015. Reproductive biology of invasive lionfish (Pterois spp.). Front. Mar. Sci., 2:7. doi:10.3389/ fmars.2015.00007

González, J., M. Grijalba-Bendeck, A. Acero P. and R. Betancur-R. 2009. The invasive red lionfish, Pterois volitans (Linnaeus, 1758), in the southwestern Caribbean Sea. Aquat. Invasions, 4(3): 507-510. DOI 10.3391/ai.2009.4.3.12

González, J.D., A. Acero P., A. Serrat-LL y R. Betancur-R. 2011. Caracterización taxonómica de la población del pez león Pterois volitans (Linnaeus 1758) (Scorpaenidae) residente en el Caribe colombiano: merística y morfometría. Biota Colomb., 12(2): 15-21.

González-Corredor, J.D., A. Acero P. y R. García-Urueña. 2016. Densidad y estructura de tallas del pez león Pterois volitans (Scorpaenidae) en el Caribe occidental insular colombiano. Bol. Invest. Mar. Cost., 45(2): 317-333.

Green, S.J. 2013. El monitoreo: una actividad fundamental. 59-82. En: J.A. Morris Jr. (ed.) El pez león invasor: guía para su control y manejo. Gulf and Caribbean Fisheries Institute Special Publication Series Number 2, Marathon, Florida, USA. 126 p.

Green, S.J. and I.M. Côté. 2009. Record densities of Indo-Pacific lionfish on Bahamian coral reefs. Coral Reefs, 28: 107. DOI 10.1007/s00338-008-0446-8

Green, S.J., J.L. Akins, A. Maljković and I.M. Côté. 2012. Invasive lionfish drive Atlantic coral reef fish declines. PLoS ONE, 7(3): e32596. doi:10.1371/journal. pone.0032596

Green, S.J., N. Tamburello, S.E. Miller, J.L. Akins and I.M. Côté. 2013. Habitat complexity and fish size affect the detection of Indo-Pacific lionfish on invaded coral reefs. Coral Reefs, 32: 413-421. DOI 10.1007/s00338-012-0987-8 
Green, S.J., E.B. Underwood and J.L. Akins. 2017. Mobilizing volunteers to sustain local suppression of a global marine invasion. Conserv. Lett., $10: 728-735$. DOI: $10.1111 /$ conl.12426

Green, S.J., E.R. Dilley, C.E. Benkwitt, A.C.D. Davis, K.E. Ingeman, T.L. Kindinger, L.J. Tuttle and M.A. Hixon. 2019. Trait-mediated foraging drives patterns of selective predation by native and invasive coral-reef fishes. Ecosphere, 10(6):e02752. 10.1002/ecs2.2752

Hackerott, S., A. Valdivia, S.J. Green, I.M. Côté, C.E. Cox, L. Akins, C.A. Layman, W.F. Precht and J.F. Bruno. 2013. Native predators do not influence invasion success of Pacific lionfish on Caribbean Reefs. PLoS ONE, 8(7): e68259. doi:10.1371/journal.pone.0068259

Hamner, R.M., D.W. Freshwater and P.E. Whitfield. 2007. Mitochondrial cytochrome $b$ analysis reveals two invasive lionfish species with strong founder effects in the western Atlantic. J. Fish. Biol., 71(Supp. B): 214-222. doi:10.1111/j.1095-8649.2007.01575.x

Hernández-Abello, J.M., R.P. García-Urueña y A. Acero P. 2015. Estructura de tallas y preferencia al sustrato del pez león (Pterois volitans) (Scorpaeniformes: Scorpaenidae) en Santa Marta (Colombia). Acta Biol. Colomb., 20(2):175-182. doi: http://dx.doi.org/10.15446/abc. v20n2.41611

Hunt, C.L., G.R. Kelly, H. Windmill, J. Curtis-Quick, H. Conlon, M.D.V. Bodmer, A.D. Rogers and D.A. Exton. 2019. Aggregating behavior in invasive Caribbean lionfish is driven by habitat complexity. Scientific Reports, 9:783. DOI:10.1038/s41598-018-37459-w

Ingeman, K.E. 2016. Lionfish cause increased mortality rates and drive local extirpation of native prey. Mar. Ecol. Prog. Ser., 558: 235-245. doi: 10.3354/ meps 11821

Lesser, M.P. and M. Slattery. 2011. Phase shift to algal dominated communities at mesophotic depths associated with lionfish (Pterois volitans) invasion on a Bahamian coral reef. Biol. Invasions, 13: 1855-1868. DOI 10.1007/s10530-011-0005-z

Morris, J.A. Jr. (Ed). 2013. El pez león invasor: guía para su control y manejo. Gulf and Caribbean Fisheries Institute Special Publication Series, No. 2, Marathon, Florida. USA. 126 p.

Muñoz, R.C., C.A. Currin and P.E. Whitfield. 2011. Diet of invasive lionfish on hard bottom reefs of the Southeast USA: insights from stomach contents and stable isotopes. Mar. Ecol. Prog. Ser., 432: 181-193. doi: 10.3354/meps09154

Muñoz-Escobar, L. y D.L. Gil-Agudelo. 2012. Composición dietaria del pez león, Pterois volitans (Pisces: Scorpaenidae), en Santa Marta y el Parque Nacional Natural Tayrona. Bol. Invest. Mar. Cost., 41(2): 471-477.

Pabón Q., P. y A. Acero P. 2015. Ecología trófica del invasor pez león Pterois volitans en el Caribe colombiano: impacto sobre familias ícticas de Santa Marta y San Andrés. 51-74. En: Campos, N.H. y A. Acero P. (eds.). 2015. Contribuciones en Ciencias del Mar de la Universidad Nacional de Colombia-2015. Cecimar, Sede Caribe, Universidad Nacional de Colombia, Santa Marta, 130 p.

Pusack, T.J., C.E. Benkwitt, K. Cure and T.L. Kindinger. 2016. Invasive red lionfish (Pterois volitans) grow faster in the Atlantic Ocean than in their native Pacific range. Environ. Biol. Fish., 99: 571-579. DOI 10.1007/s10641-016-0499-4

Rivera-Aponte, I.M., E. Hernández-Delgado, J. Bauzá-Ortega y W. Ithier-Guzmán. 2019. Distribución espacial y preferencia de hábitat del pez león Pterois volitans en el estuario urbano de la Bahía de San Juan. Perspectivas en Asuntos Ambientales, 7: 75-91.

Rojas-Vélez, S., J. Tavera and A. Acero. 2019. Unraveling lionfish invasion: is Pterois volitans truly a morphologically novel predator in the Caribbean? Bio. Invasions, 21: 1921-1931. https://doi.org/10.1007/s10530-019-01946-6

Sabido-Itzá, M.M. and M.C. García-Rivas. 2019. Record of abundance, spatial distribution and gregarious behavior of invasive lionfish Pterois spp. (Scorpaeniformes: Scorpaenidae) in coral reefs of Banco Chinchorro Biosphere Reserve, southeastern Mexico. Lat. Am. J. Aquat. Res., 47(2): $349-355$. DOI: 10.3856/vol47-issue2-fulltext-15

Sandel, V., D. Martínez-Fernández, D. Wangpraseurt and L. Sierra. 2015. Ecology and management of the invasive lionfish Pterois volitans/miles complex (Perciformes: Scorpaenidae) in Southern Costa Rica. Rev. Biol. Trop., 63(1): 213-221.

Schofield, P.J. 2009. Geographic extent and chronology of the invasion of non-native lionfish (Pterois volitans [Linnaeus 1758] and P. miles [Bennett 1828]) in the Western North Atlantic and Caribbean Sea. Aquat. Invasions, 4(3): 473-479. DOI 10.3391/ai.2009.4.3.5

Schofield, P.J. 2010. Update on geographic spread of invasive lionfishes (Pterois volitans [Linnaeus, 1758] and P. miles [Bennett, 1828]) in the Western North Atlantic Ocean, Caribbean Sea and Gulf of Mexico. Aquat. Invasions, 5(Supp1): S117-S122. doi: 10.3391/ai.2010.5.S1.024

Schmitt, E.F. and K.M. Sullivan. 1996. Analysis of a volunteer method for collecting fish presence and abundance data in the Florida Keys. Bull. Mar. Scie., 59(2): 404-416.

USGS-NAS. 2020. United States Geological Survey - Nonindigenous Aquatic Species Database (USGS-NAS). https://nas.er.usgs.gov, Access Date: 5/13/2020.

Whitfield, P.E., J.A. Hare, A.W. David, S.L. Harter, R.C. Muñoz and C.M. Addison. 2007. Abundance estimates of the Indo-Pacific lionfish Pterois volitans/ miles complex in the Western North Atlantic. Biol. Invasions, 9: 53-64. DOI 10.1007/s10530-006-9005-9 